Article

\title{
CrWSKP1, an SKP1-like Gene, Is Involved in the Self-Incompatibility Reaction of "Wuzishatangju" (Citrus reticulata Blanco)
}

\author{
Peng Li ${ }^{1, \dagger}$, Hongxia Miao ${ }^{1,2, \dagger}$, Yuewen Ma ${ }^{1}$, Lu Wang ${ }^{1}$, Guibing Hu ${ }^{1}$, Zixing Ye ${ }^{1}$, \\ Jietang Zhao ${ }^{1}$ and Yonghua Qin ${ }^{1, *}$
}

1 State Key Laboratory for Conservation and Utilization of Subtropical Agro-Bioresources/ Key Laboratory of Biology and Genetic Improvement of Horticultural Crops-South China, Ministry of Agriculture, College of Horticulture, South China Agricultural University, Guangzhou 510642, China; E-Mails: ziyue7766@163.com (P.L.); hxmrain@163.com (H.M.); pinganma@126.com (Y.M.); wxiaolu0217@163.com (L.W.); guibing@scau.edu.cn (G.H.); qyh6k@163.com (Z.Y.); zhaojietang@gmail.com (J.Z.)

2 Institute of Tropical Bioscience and Biotechnology, Chinese Academy of Tropical Agricultural Sciences/Key Laboratory of Tropical Crop Bioscience and Biotechnology, Ministry of Agriculture, Haikou 571101, China

$\dagger$ These authors contributed equally to this work.

* Author to whom correspondence should be addressed; E-Mail: qinyh@scau.edu.cn; Tel.: +86-20-8528-0505; Fax: +86-20-8528-2107.

Academic Editor: Jianhua Zhu

Received: 9 May 2015 / Accepted: 25 August 2015 / Published: 9 September 2015

\begin{abstract}
Plant S-phase kinase-associated protein 1 (SKPl) genes play crucial roles in plant development and differentiation. However, the role of SKP1 in citrus is unclear. Herein, we described a novel SKP1-like gene, designated as CrWSKP1, from "Wuzishatangju" (Citrus reticulata Blanco). The cDNA sequence of CrWSKP1 is 779 base pairs (bp) and contains an open reading frame (ORF) of $477 \mathrm{bp}$. The genomic sequence of the CrWSKP1 gene is 1296 bp with two exons and one intron. CrWSKP1 has high identity with SKP1-like genes from other plant species within two conserved regions. Approximately $85 \%$ of pollen tubes of self-pollinated CrWSKP1 transgenic tobaccos became twisted at four days after self-pollination. Pollen tube numbers of self-pollinated CrWSKP1 transformants entering into ovules were significantly fewer than that of the
\end{abstract}


control. Seed number of self-pollinated $C r W S K P 1$ transformants was significantly reduced. These results suggested that the CrWSKP1 is involved in the self-incompatibility (SI) reaction of "Wuzishatangju".

Keywords: Citrus reticulata Blanco; self-incompatibility; SKP1; genetic transformation

\section{Introduction}

Seedless fruits can be of great value for consumers and the processing industry. Self-incompatibility (SI) is an important factor that can result in seedless fruits in citrus [1-7]. SI systems are divided into sporophytic SI (SSI) and gametophytic SI (GSI). In the SSI system of Brassicaceae, S-locus cysteine-rich protein (SCR)/S-locus protein-11 (SP11) and S-locus receptor kinase (SRK) were identified to regulate a signal transduction cascade in the stigmatic papillae [8]. In the GSI system of Rosaceae, Solanaceae, and Scrophulariaceae, SI operates as a complex process involving multiple factors. GSI system can be divided into S-RNase-based SI and Papaver SI. S-RNase-based SI is mainly controlled by the pistil $S$ gene, encoding a ribonuclease (S-RNase), the pollen S-locus-encoded F-box protein (SLF/SFB) [9,10], and non-S-factors such as S-phase kinase-associated protein 1 (SKP1), S1 self-incompatibility locus-linked pollen 3.15, ubiqutitn-activating enzyme E1, and Rho-like GTPase genes [11-18]. Papaver SI is controlled by pistil S-determinant PrsS (Papaver rhoeas stigmas $S$ determinant) interacted with a $\mathrm{Ca}^{2+}$-dependent signaling network resulting in programmed cell death (PCD) of "self" pollen [19,20].

SKP1 gene encodes a small protein of approximately 160 amino acids. Plants have a large number of SKP1-like genes and they play key roles in plant cell division, growth and flower development, and protein degradation. It has been shown that the pleiotropic functions of SKP1 genes are consistent with their unique expression patterns for the respective genes in vegetative and reproductive tissues. Porat et al. [21] reported that the ATskpl gene is highly correlated with meristem activity and can be a marker for cells undergoing division in that its mRNA accumulated in all of Arabidopsis meristems and organ primordia. The SKP1-like family of Arabidopsis also exhibits a high degree of differential gene expression and gene product interaction during development [22-24]. CgSKP1, a novel SKP1 gene from "Shatian" pummelo (Citrus grandis Osbeck), may play a vital role in flower development of "Shatian" pummelo since it was highly expressed in anthers, and ovaries, but lowly in styles, while it was gradually increased during flower development of "Shatian" pummelo [25]. Hong et al. [26] isolated six SKP1-like (TaSKP) genes from common wheat (Triticum aestivum) and suggested that they may be involved in various growth and flower developmental processes according to their expression patterns. Further, Yang et al. [27] suggested that the ASK1 gene is essential for male meiosis and may control homologue separation. SKP1 and SKP1-related proteins can regulate the specific degradation of target proteins and affect the formation of other protein complexes in Arabidopsis [22].

The ubiquitination and $26 \mathrm{~S}$ proteosome system $(\mathrm{U} / 26 \mathrm{~S})$ is a major protein degradation pathway involving in plant pollen germination, pollen tube elongation and SI response. Skp1 is a core component of the SCF (Skp1, Cullin1 and F-box) complex mediating protein degradation by the U/26S. Sijacic et al. [28] demonstrated that PhSSK1 as a component of the SCF complex encodes 
pollen factors in SI reaction. In lily (Lilium longiflorum), pollen-specific SKP1-like proteins are components of functional SCF complexes and essential for pollen tube elongation in GSI lily [29]. Pollen-expressed SKP1-like genes are probably involved in the SI reaction of plants. SLF-interacting SKP1-likel (AhSSK1) is expressed specifically in pollen of Antirrhinum hispanicum and acts as an indispensable player involved in SI response, for its adaptor role of associating AhSLF with CUL1 [11,12]. Matsumoto et al. [30] identified a Skp1-like protein interacting with SFB (PavSSK1) from SI sweet cherry (Prunus avium) using a yeast two-hybrid screening against the pollen cDNA library. PavSSK1 was expressed strongly in anthers and pollen, weakly in styles, and not at all in other floral organs or leaves in sweet cherry, suggesting PavSSK1 participated in the SI reaction of sweet cherry. These results suggest that SKP1-like genes operate as complex and multiple functions in pollen tube elongation and SI reaction. However, the role of Skpl-like gene in the SI reaction of citrus is unknown.

"Wuzishatangju" (C. reticulata Blanco) is a mutant originated from self-compatible (SC, seedy) cultivar "Shatangju" [31]. It is one of the most popular mandarin cultivars in China due to its seedless, very tasty and easy-to-peel characteristics. Cytological analyses showed that the seedlessness of "Wuzishatangju" results from GSI that blocks fertilization in the ovary [1]. Our previous studies showed that expression levels of CrWSKP1 gene were significantly up-regulated in ovaries of "Wuzishatangju", and approximately 20 times higher than that of "Shatangju". The highest expression levels of CrWSKP1 gene was detected in pistils at 4 days after self-pollination of "Wuzishatangju", compared to the lowest expressions in pistils at 4 days after cross-pollination of "Wuzishatangju" $\times$ "Shatangju" [32] which is consistent with the cytological analyses [1]. We therefore hypothesized that the CrWSKP1 gene is involved in the SI reaction of "Wuzishatangju" by U/26S pathway [33]. In this study, we isolated full-length cDNA and DNA sequences of CrWSKP1 genes from SI "Wuzishatangju" mandarin based on its expressed sequence tags (ESTs) [32]. Functional characteristics of CrWSKP1 were elucidated by transgenic experiments in tobacco. The aim of this study is to explore the role of SKP1-like gene in the SI response in citrus.

\section{Results}

\subsection{Cloning and Sequence Analysis of SKP1-like Gene}

The full-length cDNA sequence of SKPl-like gene from mutant "Wuzishatangju" mandarin (designated as CrWSKP1) was $779 \mathrm{bp}$ and contained an open reading frame of 477 bp (Figure S1) encoding 158 amino acids. The ORF sequences of Skpl-like gene from its original cultivar "Shatangju" mandarin (designated as CrYSKPl) were 510 bp. Compared to "Shatangju", there were 33 bases missing in the ORF of 'Wuzishatangju' (Figure 1A). The full-length of CrWSKP1 genomic DNA from "Wuzishatangju" and "Shatangju" were, respectively, 1296 and 1294 bp with two exons and one intron (Figure 1B). There are 7 bases different in DNA sequences of CrWSKP1 and CrYSKP1 genes between "Wuzishatangju" and "Shatangju" mandarins (Figure 1C). 

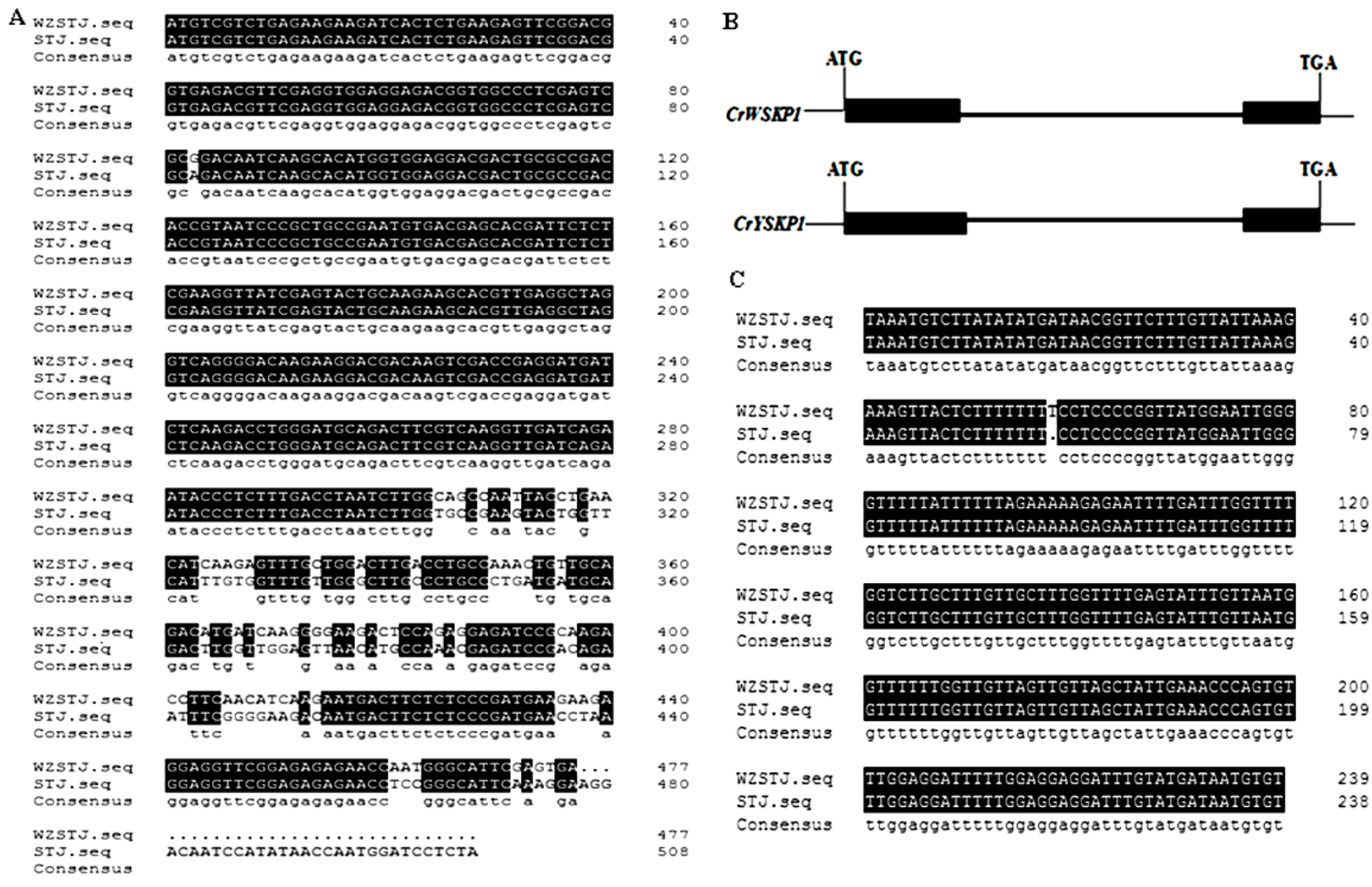

D CrKskP1

CrKSKP1
CrYSKP1

Figure 1. Analyses of $C r W S K P 1$ and $C r Y S K P 1$ genes. (A) Alignments of cDNA sequences of CrWSKP1 and CrYSKP1 genes between "Wuzishatangju" (WZSTJ) and "Shatangju" (STJ); (B) Exon-introns structure of CrWSKP1 and CrYSKP1 genes. CrWSKP1, DNA sequence of the Skpl-like gene from "Wuzishatangju"; CrYSKP1, DNA sequence of the Skpl-like gene from "Shatangju". Solid boxes indicate exons, and bold lines represent 
introns; (C) Alignments of partial DNA sequences of CrWSKP1 and CrYSKP1 genes between "Wuzishatangju" (WZSTJ) and "Shatangju" (STJ); (D) Alignments of the putative amino acid sequences of CrWSKP1 and CrYSKP1 genes from different plant species. CrWSKP1 ("Wuzishatangju”), CrYSKP1 ("Shatangju”), CzSSKP1-like (Citrus maxima, ACP20181), CaSKP1-like (Cicer arietinum, XP004512164), GmSKP1-like (Glycine max, XP003517160), VvSKP1-like (Vitis vinifera, XP002279232), AtSK20 (Arabidopsis thaliana, A8MQG7), AtSK21 (Arabidopsis thaliana, Q8LF97). Higher conserved regions were marked in box with Skp1_POZ and Skp1.

Sequence alignment showed that CrWSKP1 and CrYSKPl genes had two highly conserved regions (Skp1_POZ and Skp1) and an intervening region between the two domains (Figure 1D). CrWSKP1 shared the highest (99\%) amino acid sequence identity with CrYSKP1 gene.

\subsection{Phylogenetic Analysis of SKP1-like Genes}

To determine the phylogenetic relationship of CrWSKP1 and CrYSKP1 with SKP1-like genes from the other plants, a phylogenetic tree was constructed using 15 SKP1-like amino acids sequences (Figure 2). As shown in Figure 2, those SKP1-like genes were classified into two types, i.e., Type I and Type II. CrWSKP1 and CrYSKP1 belonged to Type I. Cluster analysis revealed that CrWSKP1 and CrYSKP1 were more closely related to the CZSKP1-like (Citrus maxima, ACP20181). CrWSKP1 shared the highest (99\%) amino acid sequence identity with CrYSKP1, followed by Citrus maxima CzSKP1-like (ACP20181) (98\%), Cicer arietinum CaSKP1-like (87\%), Glycine max GmSKP1-like (85\%), and Vitis vinifera VvSKP1-like (81\%) (Figure 2).

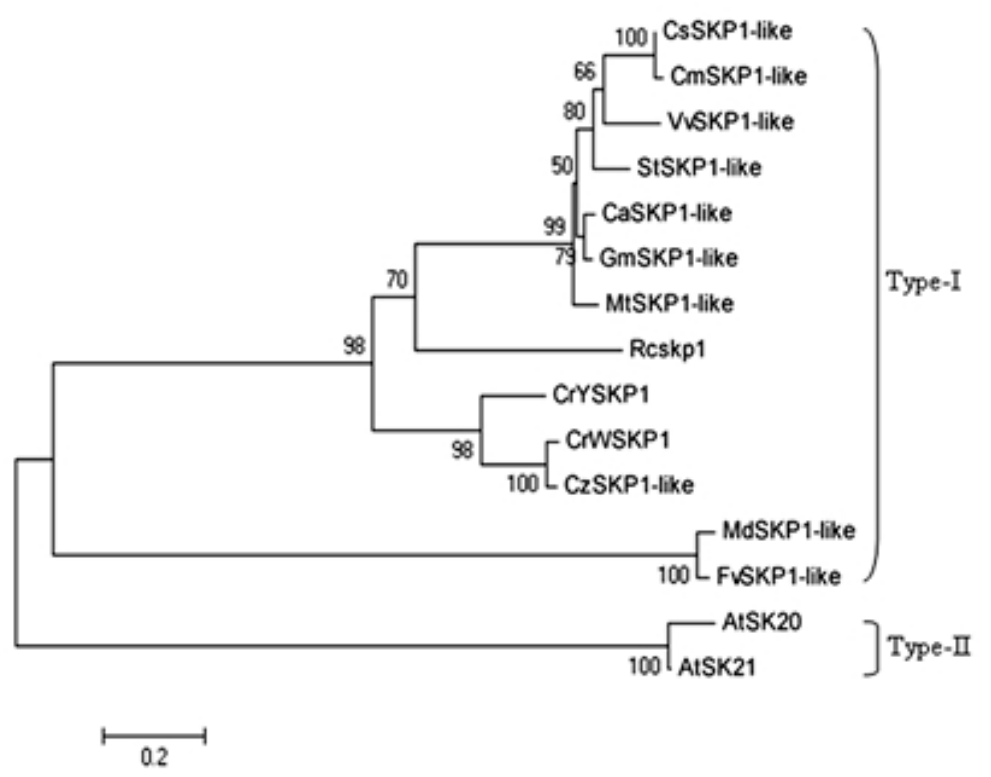

Figure 2. Phylogenetic relationship of CrWSKP1 and CrYSKP1 with SKP1-like genes from other plants. The numbers of each interior branch are the percentage bootstrap values (re-sampling). CrWSKP1 ("Wuzishatangju"), CrYSKP1 ("Shatangju"), CzSKP1-like (Citrus maxima, ACP20181), CaSKP1-like (Cicer arietinum, XP004512164), GmSKP1-like 
(Glycine max, XP003517160), VvSKPl-like (Vitis vinifera, XP002279232), CsSKP1-like (Cucumis sativus, XP004144851), CmSKPl-like (Cucumis melo, XP008447650), StSKP1-like (Solanum tuberosum, XP006362720), MtSKP1-like (Medicago truncatula, XP003612227), RcSKP1 (Ricinus communis, XP002510577), MdSKPl-like (Malus domestica, XP008357122), FvSKP1-like (Fragaria vesca subsp. Vescal (XP004287783), AtSK20 (Arabidopsis thaliana, A8MQG7), AtSK21 (Arabidopsis thaliana, Q8LF97).

\subsection{Southern Blot Analysis of SKP1-like Genes}

Southern blot was performed to determine whether SKPl-like represents a single or multi locus in the genome of "Wuzishatang" and "Shatangju" mandarins. As shown in Figure 3, multiple hybridization bands were observed when genomic DNA was digested with Hind III, EcoR I, Dra I, $X b a$ I, Sac I and Xho I, respectively. According to the result, we concluded that Skp1-like genes were encoded by a multiple-copy gene in "Wuzishatangju" and "Shatangju" genome.

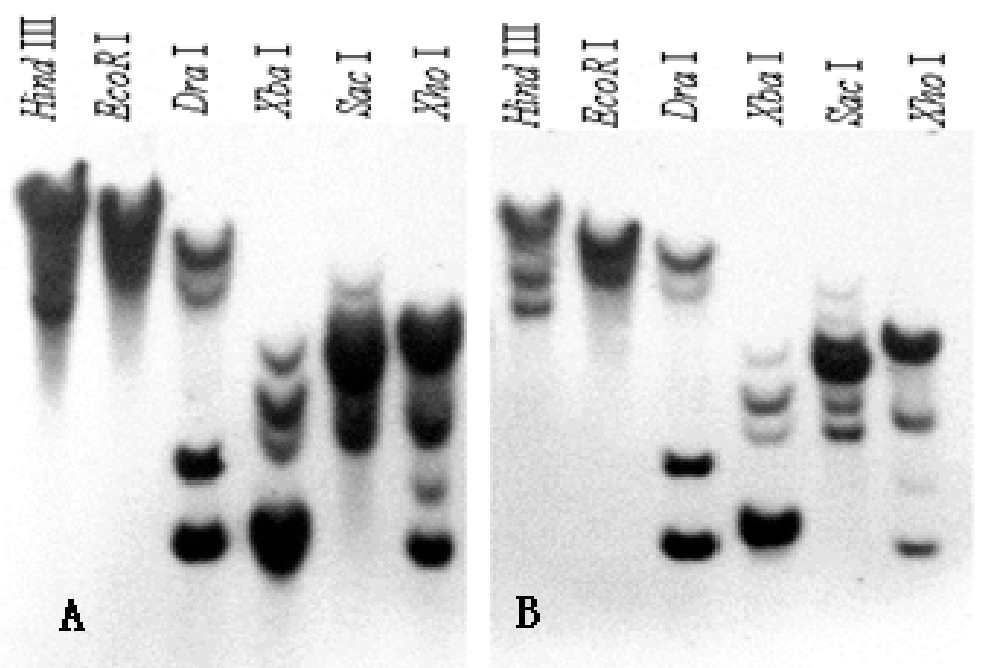

Figure 3. Southern analysis of Skpl-like genes in (A) "Wuzishatangju" and (B) "Shatangju" mandarins.

\subsection{Molecular Analyses of CrWSKP1 Transgenic Tobacco}

Tobacco transformants containing CrWSKP1 driven by CaMV 35S promoter were obtained by Agrobacterium-mediated transformation of leaf explants. The incorporation and expression of CrWSKP1 in tobacco were confirmed by PCR (Figure S2), Southern blot (Figure S3A) and real-time PCR (Figure S3B) analyses. Four independent one-copy CrWSKP1 tobacco lines were achieved (shown by red arrow) and used for further analyses. CrWSKP1 expression was detected in all transgenic tobacco compared to no expression in wild type (WT) (Figure S3B). 


\subsection{Phenotype Analyses of CrWSKP1 Transgenic Tobacco}

No significant difference in pollen viability and pollen germination rates was detected between CrWSKP1 transgenic tobacco and WT (Table S1). Three pollination combinations, namely WT $\times$ WT, $C r W S K P 1 \times C r W S K P 1, C r W S K P 1 \times W T$ were carried out to study the function of $C r W S K P 1$ gene in tobacco. After dyeing with staining agents, pollen grains became golden-yellow, pollen tubes became fluorescent green, and a bright spot of sperm and vegetative nuclei were detected at the apex of pollen tubes (Figure 4). Pollen grains germinated on the stigma at 1 day after pollination for all pollination combinations (Figure 4(A1-C1)). Pollen tubes entered into stigmas and grew downward through styles, and vascular bundles were observed around the pistillar chord at 2 days after self- and cross-pollinations (Figure 4(A2-C2)). Three days after self- and cross-pollinations, almost all pollen tubes elongated constantly in styles (Figure 4(A3-C3)). Compared to the other combinations, pollen tubes of self-pollinated $C r W S K P 1 \times C r W S K P 1$ began to twist (Figure 4(B3)). Pollen tubes of WT $\times$ WT grew normally at 4 days after self-pollination (Figure 4(A4)) while approximately $85 \%$ pollen tubes of self-pollinated CrWSKP1 transgenic tobacco became twisted at 4 days after self-pollination (Figure 4(B4)). Pollen tubes reached the bottom of style at 5 days after self- and cross-pollination for all combinations. The number of pollen tubes of self-pollinated CrWSKP $1 \times C r W S K P 1$ reaching the bottom of styles was significantly fewer than that of the other combinations (Figure 4(A5-C5)). Six days after self- and cross-pollinations, pollen tubes entered into ovaries and ovules through micropyles to complete fertilization (Figure 4(A6-C6)). Number of pollen tubes of self-pollinated CrWSKP $1 \times C r W S K P 1$ entered into ovules was significantly fewer than that of the other combinations. Compared to self-pollination of WT, seed number of transformants was significantly reduced after self-pollination of $C r W S K P 1 \times C r W S K P 1$ and significantly increased after cross-pollination of $C r W S K P 1 \times \mathrm{WT}$ (Figure 5). Those results suggested that the CrWSKPl gene is involved in the SI reaction of "Wuzishatangju".
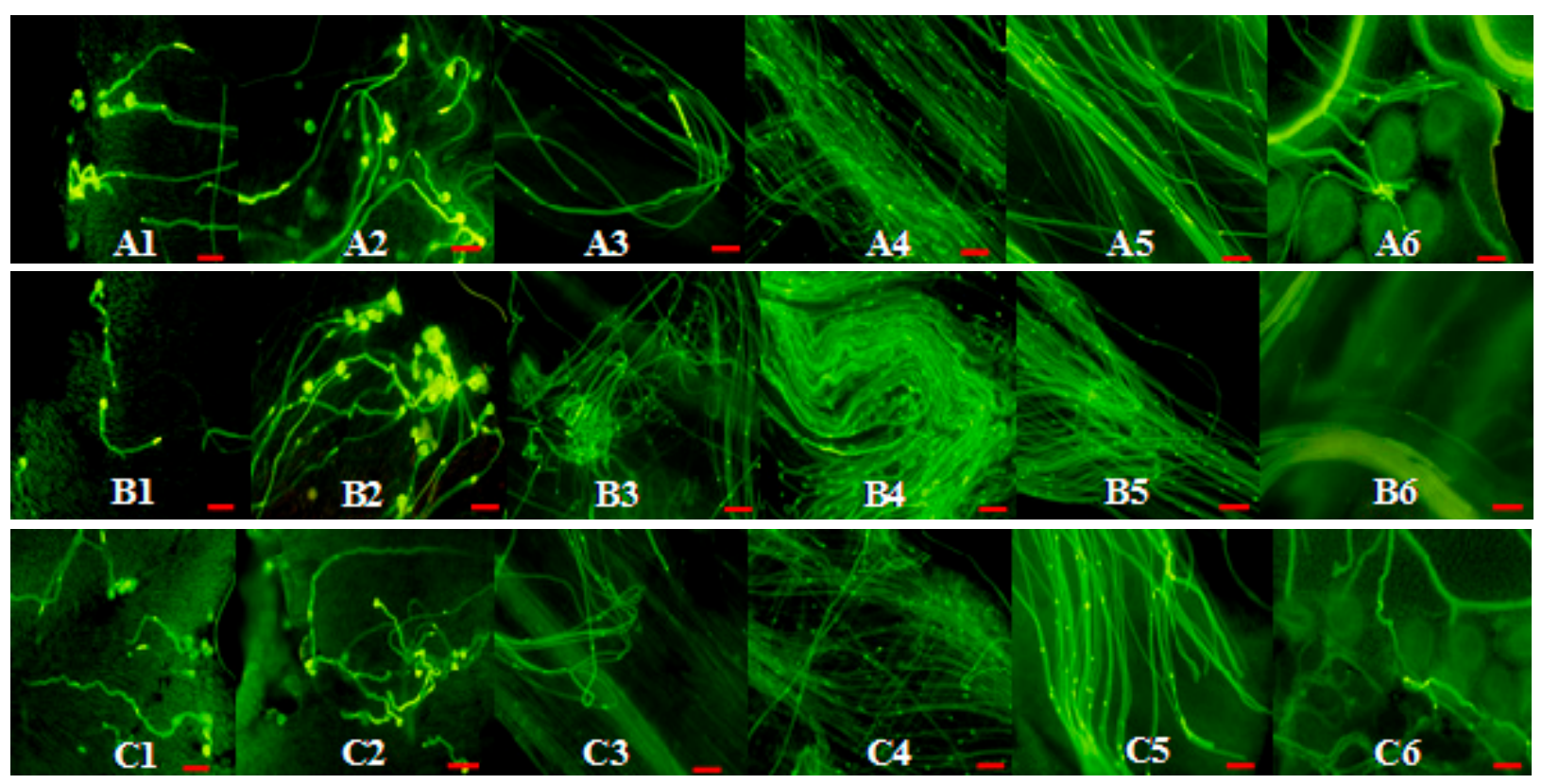

Figure 4. Pollination and fertilization of $C r W S K P 1$ transgenic tobacco at different stages $\mathrm{S}$. (A1-A6) WT $\times W T$; (B1-B6) $C r W S K P 1 \times C r W S K P 1$; (C1-C6), CrWSKP1×WT; 1, 2, 3, 4, 5, $6: 1,2,3,4,5,6$ represent days after self- and cross-pollination. Scale bar $=100 \mu \mathrm{m}$. 


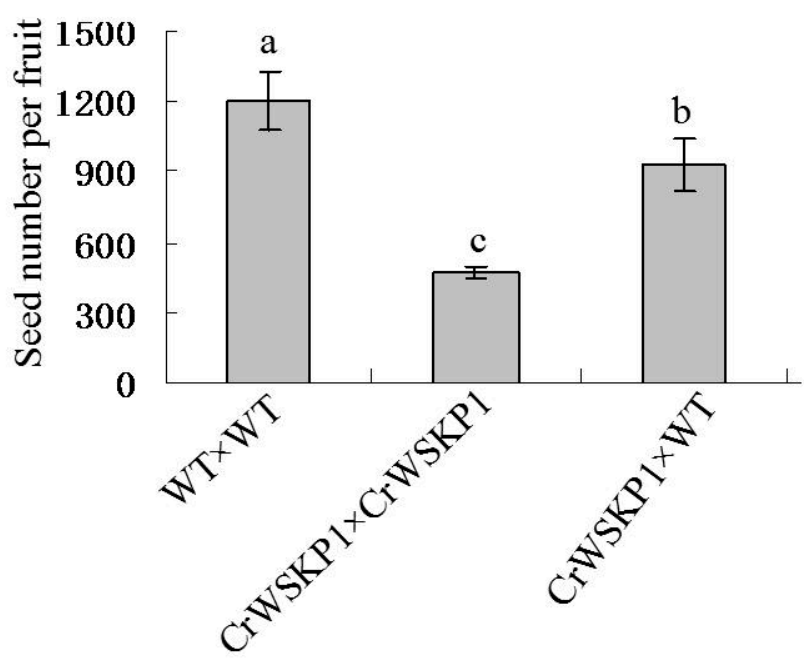

Figure 5. Seed number per fruit of CrWSKP1 transgenic tobacco after self- or cross-pollination. The vertical bars represent standard deviation $( \pm \mathrm{SD})$ of three biological replicates. a,b,c represent significant difference using the LSD test at $p \leq 0.05$.

\section{Discussions}

SKP1-like gene contains a large number of family members that play key roles in cell-cycle progression, transcriptional regulation, flower formation, signal transduction, and many other cellular processes. SKP1-like genes have been characterized from Arabidopsis [22,27,34,35], rice (Oryza sativa) [36-38], wheat (Triticum aestivum) [26], Antirrhinum hispanicum [11], lily (Lilium longiflorum) [29], "Shatian" pummelo (C. grandis Osbeck), sweet cherry (P. avium) [30] and apple (Malus $\times$ domestica) [39]. Currently, twenty-one and thirty-one SKP1-like genes were identified from Arabidopsis and Oryza sativa, respectively, and they have pleiotropic effects. However, little information is available about them in citrus. Chai et al. [25] found that $C g S K P 1$ was closely related to flower development of "Shatian" pummelo. Our previous work suggested that CrWSKP1 was obviously up-regulated in ovaries before pollination and in pistils at 4 days after self-pollination from "Wuzishatangju" [32]. In this study, full-length cDNA and DNA SKP1-like genes, designated as CrWSKP1 and CrYSKP1, were isolated from C. reticulata Blanco cv. Wuzishatangju and Shatangju. CrWSKP1 showed high amino acid sequence similarity with Shatangju (99\%), Citrus maxima (98\%), Cicer arietinum (87\%), Glycine $\max (85 \%)$ and Vitis vinifera (81\%) (Figure 2). Multiple copies of SKP1-like genes existed in the genome of both "Wuzishatangju" and "Shatangju" suggesting that SKP1-like genes belong to a multigene family.

Skp1 is a small protein of approximately 160 amino acids. It contains two highly conserved domains (a Skp1_POZ domain, which interacts with Cul1 at the $\mathrm{N}$ terminus, and a Skp1 domain, which interacts with F-box domains at the $\mathrm{C}$ terminus) and an intervening region between them $[25,30,40]$. In our study, CrWSKP1 comprised of 158 amino acids and contained both the Skp1_POZ and Skp1 domains (Figure 1C). The result is consistent with the structure and conserved domains of SKP1 protein [40]. Plant SKPI genes can be classified into type I and type II; type II is much longer than type I. In this study, deduced amino acid sequence of $C r W S K P 1$ was aligned with both type I and type II, and results showed that CrWSKP1 had high similarity with the type I of "Shatian" pummelo CgSKP1 [25], 
suggesting that CrWSKP1 belonged to type I. Genomic sequence of the CrWSKP1 gene contained two exons and one intron, a typical characteristic of type I [34,41]; a similar structure is also observed in "Shatian" pummelo [25], rice [38], and Arabidopsis [8].

Skp1 is a core component of the SCF complex mediating protein degradation by U/26S, which is involved in the SI reaction in model plants [42-46]. Kahloul et al. [38] found that these structures of SKP1 proteins seemed to interact with most of the F-box proteins. The pollen-specific AhSSK1, an Antirrhinum hispanicum SLF-interacting SKP1-like gene, could be recruited exclusively as the adaptor of putative SCF in those plants with S-RNase-based SI [11,12]. Chang et al. [29] obtained three SKP1-like genes (LSK1-LSK3) from GSI lily (Lilium longiflorum) and found that they are specifically expressed in late pollen developmental stages and the elongating pollen tube. Lily pollen tubes harboring overexpressed $L S K$ genes (LSK $\Delta$ ) were substantially inhibited within the self-pollinated styles and almost all the LSK2 $\Delta$-GFP-transformed pollen tubes were arrested on the stigma. Those results suggest that $L S K 2$ and $L S K 3$ are involved in this complex GSI machinery of lily [29]. PavSSK1, a sweet cherry (Prunus avium) SFB (PavSFB)-interacting Skpl-like gene could be a functional component of the SCF complex involved in self-/nonself-recognition in the GSI of Prunus [30]. Citrus belongs to GSI and the underlying mechanism is still unclear. In the present study, we isolated CrWSKP1 gene from SI Wuzishatangju mandarin and explored its function by transgenic experiments in tobacco. There was no significant difference in pollen viability and pollen germination rates between $C r W S K P 1$ transgenic tobacco and wild type (Table S1). Approximately 85\% of pollen tubes of self-pollinated $C r W S K P 1$ transgenic tobacco became twisted at 4 days after self-pollination and pollen tubes of self-pollinated CrWSKPI transgenic tobacco reaching the bottom of style was significantly fewer than that of wild type (Figure 4(A5-C5)). Interestingly, this disturbance was more severe in self-pollinated CrWSKPl transgenic tobacco than that in cross-pollinated pollen tubes (Figure 4(B4,C4)). Compared to self-pollination of WT, seed number of CrWSKP1 transgenic tobacco was significantly reduced whether they are self- or cross-pollination combinations (Figure 5). CrSKP1 overexpression does not cause a general defect in pollen tube growth, directionality and/or guidance. In theory, the cross $C r W S K P 1 \times \mathrm{WT}$ have the same seed number as the cross WT $\times \mathrm{WT}$. In this study, we found that some pollen tubes became twisted and could not enter ovaries at 3 days after cross-pollinated $C r W S K P 1 \times \mathrm{WT}$. Therefore, cross $C r W S K P 1 \times \mathrm{WT}$ does not have the same seed number than that of WT $\times$ WT (Figure 5). However, our results did not clarify whether CrWSKP1 is the cause of SI reaction of "Wuzishatangju" through the SCF complex mediating protein degradation by U/26S. Further work on identifying protein interacting with CrWSKP1 is necessary to elucidate its crucial role in the SI reaction of "Wuzishatangju".

\section{Experimental Section}

\subsection{Plant Materials}

Mutant "Wuzishatangju" (SI) and its original cultivar "Shatangju" (SC) mandarins were grown in the same orchard of South China Agricultural University and used as plant materials. Leaves from aseptic tobacco (Nicotiana tabacum L. cv. W38) plantlets cultured on MS medium [47] were used as the source of transformation explants. 


\subsection{Genomic DNA and Total RNA Extraction}

Genomic DNA was isolated using a CTAB method [48]. Total RNA was extracted using the RNAout kit (TIANDZ, Beijing, China), and pretreated by RNase-free DNase I (TaKaRa, Dalian, China). Qualities and concentrations of RNA and DNA were determined using a spectrophotometer (Bio-Rad Laboratories, Hercules, CA, USA) and 1.2\% (w/v) agarose gel electrophoresis. The first strand cDNA was synthesized according to the manufacturer's instructions using a cDNA synthesis kit (TaKaRa).

\subsection{Cloning and Sequence Analysis of Skp1-like Gene}

A SKPl-like coding sequence (CDS) was obtained through SSH libraries of $72 \mathrm{~h}$ styles after self-pollination of "Wuzishatangju" and cross-pollination of "Wuzishatangju" $\times$ "Shatangju" [32]. A pair of primers (SKP1-F and SKP1-R) (Table 1) was used to amplify the full-length DNA and cDNA of CrWSKP1 and $C r Y S K P 1$ genes including start and stop codons.

Table 1. Primer sequences and PCR procedures used in this study.

\begin{tabular}{lll}
\hline Primer Name & Primer Sequences $\left(5^{\prime}-\mathbf{3}^{\prime}\right)$ & Procedures of PCR \\
\hline SKP1-F & GAAACGATGTCGTCTGAGAAGAAGAT & $94{ }^{\circ} \mathrm{C} 4 \mathrm{~min} ; 94{ }^{\circ} \mathrm{C} 30 \mathrm{~s}, 59{ }^{\circ} \mathrm{C} 30 \mathrm{~s}$, \\
SKP1-R & GTCCTTCACTCGAATGCCCATTGGTT & $72{ }^{\circ} \mathrm{C} 60 \mathrm{~s}, 35$ cycles; $72{ }^{\circ} \mathrm{C} 10 \mathrm{~min}$ \\
\hline TZSKP1-F & ATAAAATGAGAACTTAATTTAC & $94{ }^{\circ} \mathrm{C} 4 \mathrm{~min} ; 94{ }^{\circ} \mathrm{C} 30 \mathrm{~s}, 57{ }^{\circ} \mathrm{C} 30 \mathrm{~s}$, \\
TZSKP1-R & TCACTCGAATGCCCATTGGTT & $72{ }^{\circ} \mathrm{C} 60 \mathrm{~s}, 35$ cycles; $72{ }^{\circ} \mathrm{C} 10 \mathrm{~min}$ \\
\hline NPTII-F & GTTCTTTTTGTCAAGACCGACC & $94{ }^{\circ} \mathrm{C} 4 \mathrm{~min} ; 94{ }^{\circ} \mathrm{C} 30 \mathrm{~s}, 55^{\circ} \mathrm{C} 30 \mathrm{~s}$, \\
NPTII-R & CAAGCTCTTCAGCAATATCACG & $72{ }^{\circ} \mathrm{C} 60 \mathrm{~s}, 35$ cycles; $72{ }^{\circ} \mathrm{C} 10 \mathrm{~min}$ \\
\hline 35S-F & GAGGACCTAACAGAACTCG & $94{ }^{\circ} \mathrm{C} 4 \mathrm{~min} ; 94{ }^{\circ} \mathrm{C} 30 \mathrm{~s}, 57{ }^{\circ} \mathrm{C} 30 \mathrm{~s}$, \\
35S-R & GTCTTGCGAAGGATAGTGG & $72{ }^{\circ} \mathrm{C} 60 \mathrm{~s}, 35 \mathrm{cycles} ; 72{ }^{\circ} \mathrm{C} 10 \mathrm{~min}$ \\
\hline RTSKP1-F & ATGTCGTCTGAGAAGAAGATC & $94{ }^{\circ} \mathrm{C} 5 \mathrm{~min} ; 94{ }^{\circ} \mathrm{C} 30 \mathrm{~s}, 59{ }^{\circ} \mathrm{C} 30 \mathrm{~s}$, \\
RTSKP1-R & CTCGAATGCCCATTGGTTCTC & $72{ }^{\circ} \mathrm{C} 1 \mathrm{~min}, 35 \mathrm{cycles} ; 72{ }^{\circ} \mathrm{C} 10 \mathrm{~min}$ \\
\hline ChvA-F & TCCATCAGCAACGTGTCGGTGCT & $94{ }^{\circ} \mathrm{C} 4 \mathrm{~min} ; 94{ }^{\circ} \mathrm{C} 30 \mathrm{~s}, 60{ }^{\circ} \mathrm{C} 30 \mathrm{~s}$, \\
ChvA-R & GTGGAAAGGCGGTGAGCGATGAT & $72{ }^{\circ} \mathrm{C} 90 \mathrm{~s}, 35$ cycles; $72{ }^{\circ} \mathrm{C} 10 \mathrm{~min}$ \\
\hline QSKP1-F & CTCTTTGACCTAATCTTGGCAG & $95{ }^{\circ} \mathrm{C} 1 \mathrm{~min} ; 95{ }^{\circ} \mathrm{C} 15 \mathrm{~s}, 55^{\circ} \mathrm{C} 20 \mathrm{~s}$, \\
QSKP1-R & CTTGCGGATCTCCTCTGGAGTC & $72{ }^{\circ} \mathrm{C} 30 \mathrm{~s}, 40$ cycles \\
\hline Q-actin-F & CTGGCATTGCAGATCGTATGA & $95{ }^{\circ} \mathrm{C} 1 \mathrm{~min} ; 95{ }^{\circ} \mathrm{C} 15 \mathrm{~s}, 55{ }^{\circ} \mathrm{C} 20 \mathrm{~s}$, \\
Q-actin-R & GCGCCACCACCTTGATCTT & $72{ }^{\circ} \mathrm{C} 30 \mathrm{~s}, 40$ cycles \\
\hline
\end{tabular}

Nucleotide sequences of CrWSKP1 and CrYSKP1 genes were analyzed using the NCBI Blast program [49]. Amino acid sequence analysis and a homology tree were constructed using MEGA software (Version 5.05) (Arizona State University, Tempe, AZ, USA). The number for each interior branch is the percent bootstrap values calculated from 1000 replicates.

\subsection{Southern Blot Analysis}

Genomic DNA (10.0 $\mu \mathrm{g}$ per sample) from "Wuzishatangju" and "Shatangju" mandarins was digested in separate tubes with Hind III, EcoR I, Dra I, Xba I, Sac I and Xho I overnight at $37{ }^{\circ} \mathrm{C}$, separated on a $0.8 \%(w / v)$ agarose gel and transferred onto Hybond- $\mathrm{N}^{+}$nylon membranes $\left(\right.$Hybond $\mathrm{N}^{+}$, Amersham Biosciences Corp, NJ, USA) [50]. Probes (353 bp) from a partial region of the CrWSKP1 
gene for hybridization were prepared from PCR product using TZSKP1-F and TZSKP1-R primers (Table 1) and used in DIG-dUTP according to the manufacturer's instruction (Roche Applied Science, Mannheim, Germany). Pre-hybridization, hybridization, and detection of the labeled probe were performed according to Miao et al. [51].

\subsection{Genetic Transformation of Tobacco}

To construct the CrWSKP1 expression vector, the CrWSKPI fragments were excised using BamH I and $S a c$ I and inserted into pBI121 vector digested with the same two enzymes. The constructed expression vector was named pBI121-CrWSKP1 under control of the CaMV 35S promoter. The recombinant plasmids were transferred into Agrobacterium tumefaciens strain EHA105 by the liquid nitrogen freeze-thaw method.

Putative transformants were screened by PCR analysis using NPT II, 35S, RTSKP1 and ChvA primers (Table 1). $\mathrm{T}_{0}$ positive-PCR transformed lines were further confirmed by Southern blot. For Southern hybridization, $20.0 \mu \mathrm{g}$ of genomic DNA was digested with EcoR I (cloning site for CrWSKP1 gene in pBI121-CrWSKP1 vector), blotted, and probed by using the NPT II gene according to the protocol of Miao et al. [51].

Expression levels of CrWSKP1 in single-copy $\mathrm{T}_{0}$ transgenic lines were assayed by real-time PCR using the SYBR ExScript RT-PCR Kit (TaKaRa). Q-Actin-F and Q-Actin-R primers were used to amplify the tobacco actin gene (accession No. U60491.1) as a loading control to normalize samples in separate tubes. Quantitative real-time PCR were performed in triplicate for each sample using the primers of QSKP1-F and QSKP1-R (Table 1). The expression levels of the CrWSKP1 gene were calculated using the $2^{-\Delta \Delta C t}$ method [52].

\subsection{Pollination and Fertilization of Transgenic Tobacco}

To explore function of $C r W S K P 1$ gene in transgenic tobacco, mature pollen grains from single-copy transgenic lines and wild types (WT) were collected to assay pollen viability and pollen germination rate according to Ye et al. [1]. Before artificial-pollination, the flowers were emasculated. Pollen was gently smudged with a brush on the stigma and then quickly bagged. Pistils, including stigmas, styles and ovaries, were collected at 1,2,3,4,5 and 6 days from single-copy self-pollinated transgenic CrWSKP1 lines $\times$ transgenic $C r W S K P 1$ lines $(C r W S K P 1 \times C r W S K P 1)$, cross-pollinated transgenic $C r W S K P 1$ lines $\times \mathrm{WT}(C r W S K P 1 \times \mathrm{WT})$ using self-pollinated $\mathrm{WT} \times \mathrm{WT}$ as control. Sections were made following the procedures of Shi and Hou [53] and Tao et al. [54]. Pollen tubes were stained with 0.1\% aniline blue (Sigma, St. Louis, MO, USA) for $30 \mathrm{~min}$. Pollination and fertilization of all combinations were observed with a fluorescent microscope (OLYMPUS BH $\mathrm{BH}_{2}$ RFA, Olympus Optical Co., Ltd., Tokyo, Japan) and photos were obtained by a digital microphotograph system (OLYMPUS DP70, Olympus Optical Co., Ltd., Tokyo, Japan). Mature seed number per fruit of CrWSKP1 transgenic tobacco was collected after self- or cross-pollination combinations. 


\subsection{Statistical Analysis}

Statistical analyses were performed using DPS 7.05 software (Zhejiang University, Hangzhou, Zhejiang, China). Differences between compared sets were considered significantly using the LSD test at $p \leq 0.05$.

\section{Conclusions}

We cloned a CrWSKP1 gene from "Wuzishatangju" mandarin with high similarity to the other plant SKP1-like genes. Approximately $85 \%$ of pollen tubes of self-pollinated CrWSKP1 transgenic tobacco plants became twisted at 4 days after self-pollination. Pollen tubes of self-pollinated CrWSKP1 transformants entering into ovule were significantly fewer than that of the control. Seed number of self-pollinated CrWSKP1 transformants was significantly reduced. Those results suggested that the CrWSKP1 gene is involved in the SI reaction of "Wuzishatangju". Further work using yeast two-hybrid analysis is needed to identify proteins interacting with CrWSKP1 and to then study their in vivo function in citrus or other model plant systems.

\section{Supplementary Materials}

Supplementary materials can be found at http://www.mdpi.com/1422-0067/16/09/21695/s1.

\section{Acknowledgments}

This work was supported by National Natural Science Foundation of China (No. 31000899, 31471858 and 31401843), Guangdong Province Science Foundation of China (No. S2013020013084 and S2013010011950), Research Fund for the Doctoral Program of Higher Education of China (No. 20104404120015 and 20114404110018), Foundation for Higher Education Discipline and Specialty Construction of Guangdong Provincial Department of Education (2013KJCX0031), Science and Technology Planning Project of Guangzhou (2010r1-C771), the Open Foundation of State Key Laboratory for Conservation and Utilization of Subtropical Agro-bioresources, South China Agricultural University (No. KSL-CUSAb-2012-09), Key Laboratory of Innovation and Utilization for Germplasm Resources in Horticultural Crops in Southern China of Guangdong Higher Education Institutes, South China Agricultural University (No. KBL11008).

\section{Author Contributions}

Conceived and designed the experiments: Yonghua Qin. Performed the experiments: Peng Li, Hongxia Miao and Yuewen Ma. Analyzed the data: Peng Li, Hongxia Miao and Yonghua Qin. Contributed reagents/materials/analysis tools: Lu Wang, Guibing $\mathrm{Hu}$, Zixing Ye and Jietang Zhao. Wrote the paper: Peng Li, Hongxia Miao and Yonghua Qin.

\section{Conflicts of Interest}

The authors declare no conflict of interest. 


\section{References}

1. Ye, W.J.; Qin, Y.H.; Ye, Z.X.; Teixeira da Silva, J.A.; Zhang, L.X.; Wu, X.Y.; Lin, S.Q.; Hu, G.B. Seedless mechanism of a new mandarin cultivar "Wuzishatangju" (Citrus reticulata Blanco). Plant Sci. 2009, 177, 19-27.

2. Distefano, G.; Caruso, M.; Malfa, S.L.; Gentile, A.; Tribulato, E. Histological and molecular analysis of pollen-pistil interaction in clementine. Plant Cell Rep. 2009, 28, 1439-1451.

3. Ngo, B.X.; Wakana, A.; Kim, J.H.; Mori, T.; Sakai, K. Estimation of self-incompatibility S genotypes of Citrus cultivars and plants based on controlled pollination with restricted number of pollen grains. J. Fac. Agric. Kyushu Univ. 2010, 55, 67-72.

4. Caruso, M.; Merelo, P.; Distefano, G.; La Malfa, S.; Lo Piero, A.R.; Tadeo, F.R.; Talon, M.; Gentile, A. Comparative transcriptome analysis of stylar canal cells identifies novel candidate genes implicated in the self-incompatibility response of Citrus clementina. BMC Plant Biol. 2012, 1, doi:10.1186/1471-2229-12-20.

5. Mesejo, C.; Yuste, R.; Martínez-Fuentes, A.; Reig, C.; Iglesias, D.J.; Primo-Millo, E.; Agustí, M. Self-pollination and parthenocarpic ability in developing ovaries of self-incompatible Clementine mandarins (Citrus clementina). Physiol. Plant. 2013, 148, 87-96.

6. Zhang, S.W.; Ding, F.; He, X.H.; Luo, C.; Huang, G.X.; Hu, Y. Characterization of the 'Xiangshui' lemon transcriptome by de novo assembly to discover genes associated with self-incompatibility. Mol. Genet. Genom. 2015, 290, 365-375.

7. Zhang, S.W.; Huang, G.X.; Ding, F.; He, X.H.; Pan, J.C. Mechanism of seedlessness in a new lemon cultivar "Xiangshui" (Citrus limon (L.) Burm. F.). Sex. Plant Reprod. 2012, 25, 337-345.

8. Takayama, S.; Shimosato, H.; Shiba, H.; Funato, M.; Che, F.S.; Watanabe, M.; Iwano, M.; Isogai, A. Direct ligand-receptor complex interaction controls Brassica self-incompatibility. Nature 2001, 413, 534-538

9. Sassa, H.; Kakui, H.; Minamikawa, M. Pollen-expressed F-box gene family and mechanism of S-RNase-based gametophytic self-incompatibility (GSI) in Rosaceae. Sex. Plant Reprod. 2010, 23, $39-43$.

10. Sun, P.; Kao, T.H. Self-incompatibility in Petunia inflata: The relationship between a self-incompatibility locus F-box protein and its non-self S-RNases. Plant Cell 2013, 25, 470-485.

11. Huang, J.; Zhao, L.; Yang, Q.Y.; Xue, Y.B. AhSSK1, a novel SKP1-like protein that interacts with the S-locus F-box protein SLF. Plant J. 2006, 46, 780-793.

12. Zhao, L.; Huang, J.; Zhao, Z.H.; Li, Q.; Sims, T.L.; Xue, Y.B. The Skp1-like protein SSK1 is required for cross-pollen compatibility in S-RNase-based self-incompatibility. Plant J. 2010, 62, $52-63$.

13. Miao, H.X.; Ye, Z.X.; Qin, Y.H.; Hu, G.B. Molecular characterization and expression analysis of S1 self-incompatibility locus-linked pollen 3.15 gene in Citrus reticulata. J. Integr. Plant Biol. 2013, 55, 443-452.

14. Miao, H.X.; Ye, Z.X.; Qin, Y.H.; Hu, G.B. Molecular characterization and expression analysis of ubiqutitn-activating enzyme E1 gene in Citrus reticulata. Gene 2013, 513, 249-259. 
15. Miao, H.X.; Ye, Z.X.; Qin, Y.H.; Hu, G.B. Identifying differentially expressed genes in pollen from self-incompatible "Wuzishatangju" and self-compatible "Shatangju" mandarins. Int. J. Mol. Sci. 2013, 14, 8538-8555.

16. Yuan, H.; Meng, D.; Gu, Z.Y.; Li, W.; Wang, A.D.; Yang, Q.; Zhu, Y.D.; Li, T.Z. A novel gene, $M d S S K 1$, as a component of the SCF complex rather than MdSBP1 can mediate the ubiquitination of S-RNase in apple. J. Exp. Bot. 2014, 65, 3121-3131.

17. Meng, D.; Gu, Z.Y.; Li, W.; Wang, A.D.; Yuan, H.; Yang, Q.; Li, T.Z. Apple MdABCF assists in the transportation of S-RNase into pollen tubes. Plant J. 2014, 78, 990-1002.

18. Meng, D.; Gu, Z.Y.; Wang, A.D.; Yuan, H.; Li, W.; Yang, Q.; Duan, X.W.; Li, T.Z. Screening and characterization of apple Rho-like GTPase (MdROPs) genes related to S-RNase mediated self-incompatibility. Plant Cell Tissue Organ Cult. 2014, 117, 465-476.

19. Wheeler, M.J.; Armstrong, S.A.; Franklin-Tong, V.E.; Franklin, F.C.H. Genomic organization of the Papaver rhoeas self-incompatibility $S_{l}$ locus. J. Exp. Bot. 2003, 54, 131-139.

20. Thomas, S.G.; Franklin-Tong, V.E. Self-incompatibility triggers programmed cell death in Papaver pollen. Nature 2004, 429, 305-309.

21. Porat, R.; Lu, P.; O’Neill, S.D. Arabidopsis SKP1, a homologue of a cell cycle regulator gene, is predominantly expressed in meristematic cells. Planta 1998, 204, 345-351.

22. Takahashi, N.; Kuroda, H.; Kuromori, T.; Hirayama, T.; Seki, M.; Shinozaki, K.; Shimada, H.; Matsui, M. Expression and interaction analysis of Arabidopsis Skp1-related genes. Plant Cell Physiol. 2004, 45, 83-91.

23. Dezfulian, M.H.; Soulliere, D.M.; Dhaliwal, R.K.; Sareen, M.; Crosby, W.L. The SKPl-like gene family of Arabidopsis exhibits a high degree of differential gene expression and gene product interaction during development. PLoS ONE 2012, 7, e50984.

24. Zhao, D.Z.; Ni, W.M.; Feng, B.M.; Han, T.F.; Petrasek, M.G.; Ma, H. Members of the Arabidopsis-SKP1-like gene family exhibit a variety of expression patterns and may play diverse roles in Arabidopsis. Plant Physiol. 2003, 133, 203-217.

25. Chai, L.J.; Biswas, M.K.; Ge, X.X.; Deng, X.X. Isolation, characterization, and expression analysis of an SKP1-like gene from "Shatian" Pummelo (Citrus grandis Osbeck). Plant Mol. Biol. Rep. 2010, 28, 569-577.

26. Hong, M.J.; Kim, D.Y.; Seo, Y.W. SKPl-like-related genes interact with various F-box proteins and may form SCF complexes with Cullin-F-box proteins in wheat. Mol. Biol. Rep. 2013, 40, 969-981.

27. Yang, M.; Hu, Y.; Lodhi, M. ; McCombie, W.R.; Ma, H. The Arabidopsis SKP1-LIKE1 gene is essential for male meiosis and may control homologue separation. Proc. Natl. Acad. Sci. USA 1999, 96, 11416-11421.

28. Sijacic, P.; Wang, X.; Skirpan, A.L.; Wang, Y.; Dowd, P.E.; McCubbin, A.G.; Huang, S.; Kao, T.H. Identification of the pollen determinant of S-RNase-mediated self-incompatibility. Nature 2004, 429, 302-305.

29. Chang, L.C.; Guo, C.L.; Lin, Y.S.; Fu, H.; Wang, C.S.; Jauh, G.Y. Pollen-specific SKP1-like proteins are components of functional SCF complexes and essential for lily pollen tube elongation. Plant Cell Physiol. 2009, 50, 1558-1572. 
30. Matsumoto, D.; Yamane, H.; Abe, K.; Tao, R. Identification of a Skp1-like protein interaction with SFB, the pollen $S$ determinant of the gametophytic self-incompatibility in Prunus. Plant Physiol. 2012, 159, 1252-1262.

31. Ye, Z.X.; Zeng, T.; Xu, J.K.; Luo, Z.D.; Hu, G.B.; Zhang, Z.Q.; Ji, Z.L.; Chen, Y.C.; Chen, G.L.; Chen, L.L.; et al. Wuzishatangju, a new mandarin cultivar (in Chinese). J. Fruit Sci. 2006, 23, 149-150.

32. Miao, H.X.; Ye, Z.X.; Qin, Y.H.; Hu, G.B. Identification of differentially expressed genes in 72 h styles from self-incompatible Citrus reticulata. Sci. Hortic. 2013, 161, 278-285.

33. Miao, H.X.; Ye, Z.X.; Hu, G.B.; Qin, Y.H. Comparative transcript profiling of gene expression between self-incompatibility and self-compatibility mandarins by suppression subtractive hybridization and cDNA microarray. Mol. Breed. 2015, 35, doi:10.1007/s11032-015-0204-x.

34. Risseeuw, E.P.; Daskalchuk, T.E.; Banks, T.W.; Liu, E.; Cotelesage, J.L.; Hellmann, H.; Estelle, M.; Somers, D.E.; William, L. Protein interaction analysis of SCF ubiquitin E3 ligase subunits from Arabidopsis. Plant J. 2003, 34, 753-767.

35. Yang, X.; Timofejeva, L.; Ma, H.; Makaroff, C. The Arabidopsis SKP1 homolog ASK1 controls meiotic chromosome remodeling and release of chromatin from the nuclear membrane and nucleolus. J. Cell Sci. 2006, 119, 3754-3763.

36. Li, C.J.; Liang, Y.; Chen, C.B.; Li, J.H.; Xu, Y.Y.; Xu, Z.H.; Ma, H.; Chong, K. Cloning and expression analysis of TSK1, a wheat SKP1 homologue,and functional comparison with Arabidopsis ASK1 in male meiosis and auxin signaling. Funct. Plant Biol. 2006, 33, 381-390.

37. Kong, H.; Landherr, L.L.; Frohlich, M.W.; Leebens-Mack, J.; Ma, H.; de Pamphilis, C.W. Patterns of gene duplication in the plant SKPl gene family in angiosperms: Evidence for multiple mechanisms of rapid gene birth. Plant J. 2007, 50, 873-885.

38. Kahloul, E.; HajSalah, E.B.; Boulaflous, A.; Ferchichi, A.; Kong, H.; Mouzeyar, S.; Bouzidi, M.F. Structural, expression and interaction analysis of rice SKPl-like genes. DNA Res. 2013, 20, $67-78$.

39. Minamikawa, M.F.; Koyano, R.; Kikuchi, S.; Koba, T.; Sassa, H. Identification of SFBB-containing canonical and noncanonical SCF complexes in pollen of apple (Malus $\times$ domestica). PLoS ONE 2014, 9, e97642.

40. Schulman, B.A.; Carrano, A.C.; Jeffrey, P.D.; Bowen, Z.; Kinnucan, E.R.; Finnin, M.S.; Elledge, S.J.; Harper, J.W.; Pagano, M.; Pavletich, N.P. Insights into SCF ubiquitin ligases from the structure of the Skp1-Skp2 complex. Nature 2000, 408, 381-386.

41. Kong, H.; Leebens-Mack, J.; Ni, W.; de Pamphilis, C.W.; Ma, H. Highly heterogeneous rates of evolution in the SKP1 gene family in plants and animals: Functional and evolutionary implications. Mol. Biol. Evol. 2004, 21, 117-128.

42. Moon, J.; Parry, G.; Estelle, M. The ubiquitin-proteasome pathway and plant development. Plant Cell 2004, 16, 3181-3195.

43. Qiao, H.; Wang, H.; Zhao, L.; Zhou, J.L.; Huang, J.; Zhang, Y.S.; Xue, Y.B. The F-box protein AhSLF-S physically interacts with S-RNases that may be inhibited by the ubiquitin/26S proteasome pathway of protein degradation during compatible pollination in Antirrhinum. Plant Cell 2004, 16, $582-595$. 
44. Yu, X.M.; Lan, X.G.; Li, Y.H. The Ub/26S proteasome pathway and self-incompatible responses in flowering plants (in Chinese). Chin. Bull. Bot. 2006, 23, 197-206.

45. Zhang, Y.J.; Zhao, Z.H.; Xue, Y.B. Roles of proteolysis in plant self-incompatibility. Annu. Rev. Plant Biol. 2009, 60, 21-42.

46. Chen, G.; Zhang, B.; Liu, L.J.; Li, Q.; Zhang, Y.E.; Xie, Q.; Xue, Y.B. Identification of a ubiquitin-binding structure in the S-locus F-Box protein controlling S-RNase-based self-incompatibility. J. Genet. Genom. 2012, 39, 93-102.

47. Murashige, T.; Skoog, F. A revised medium for rapid growth and bio assays with tobacco cultures. Physiol. Plant. 1962, 15, 473-497.

48. Song, G.L.; Cui, R.X.; Wang, K.B.; Guo, L.P.; Li, S.H. A rapid improved CTAB method for extraction of cotton genomic DNA (in Chinese). Acta Gossypii Sin. 1998, 10, 273-275.

49. The National Center for Biotechnology Information. Available online: http://www.ncbi.nlm.nih.gov/ (accessed on 25 January 2014).

50. Sambrook, J.F.; Russell, D.W. Molecular Cloning: A Laboratory Manual, 3rd ed.; Cold Spring Harbor Laboratory Press: New York, NY, USA, 2001; pp. 474-490.

51. Miao, H.X.; Qin Y.H.; Teixeira da Silva, J.A.; Ye, Z.X.; Hu, G.B. Cloning and expression analysis of $S$-RNase homologous gene in Citrus reticulate Blanco cv. Wuzishatangju. Plant Sci. 2011, 180, 358-367.

52. Livak, K.J.; Schmittgen, T.D. Analysis of relative gene expression data using real-time quantitative PCR and the $2^{-\triangle \Delta C t}$ method. Methods 2001, 25, 402-408.

53. Shi, G.J.; Hou, X.L. Measurement of self-incompatible by fluoroscope observation in non-heading Chinese cabbage (in Chinese). J. Wuhan Bot. Res. 2004, 22, 197-200.

54. Tao, S.T.; Zhang, S.L.; Chen, D.X.; Wei, B.Y. Study on characteristics of in situ pollen germination and tube growth of Prunus mume (in Chinese). J. Fruit Sci. 2004, 21, 338-340.

(C) 2015 by the authors; licensee MDPI, Basel, Switzerland. This article is an open access article distributed under the terms and conditions of the Creative Commons Attribution license (http://creativecommons.org/licenses/by/4.0/). 